read with interest the commentary by Doust in the 2004 Sep/Oct issue. ${ }^{1}$ I concur that the laboratory evaluation of vaginitis, in general, is superior to individual historical and physical findings. However, 3 additional points deserve emphasis. Firstly, evaluating combinations of clinical findings for calculating likelihood ratios (LRs) would be helpful, as combinations have been evaluated in at least 1 other clinical entity. ${ }^{2}$ Secondly, evaluating LRs for previous use of antibiotics may prove useful, since they can predispose to vaginal candidiasis (VC). Thirdly, I would like to emphasise the value of the lack of odour in bacterial vaginosis (BV) and lack of yellow discharge in vaginal trichomoniasis (VT), as the negative LRs are 0.07 and 0.12 , respectively. A physician could make the decision to treat a woman empirically for vaginal candidiasis with over-the-counter intravaginal miconazole without performing an expensive laboratory examination, as long as there was no history of odorous discharge (BV) or yellow discharge (VT). For example, based on the

\section{Author response}

D Nardone is correct about the negative predictive value of the symptom of lack of odour for bacterial vaginosis. He is also correct that lack of yellow discharge makes trichomoniasis less likely, but this is only when seen on physical examination, and this conclusion is based on only 1 study of 9 patients. Furthermore, it is not clear that there is value to the lack of yellow discharge as a symptom in the diagnosis of trichomoniasis.

\section{Correction}

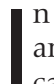
the commentary for the abstract "Review: vaginal signs and symptoms perform poorly in diagnosing vaginal candidiasis, bacterial vaginosis, and vaginal trichomoniasis, ${ }^{\prime \prime}$ the second sentence of the second paragraph should be "The absence of symptoms and signs is even less helpful in ruling out disease, with only the absence of odour ruling out $\mathrm{BV}$, and absence of yellow discharge on physical examination ruling out VT." The original sentence was as follows: "The prevalence of BV (46\%) and VT $(12 \%),{ }^{3}$ the post-test probabilities for the absences of odour and yellow discharge are $6.0 \%(\mathrm{BV})$ and $2 \%(\mathrm{VT})$. Neither probability is likely to be above one's treatment threshold. Only if the miconazole therapy has failed and the patient has persistent or recurrent external genital symptoms would laboratory examinations be performed.

DAVID A. NARDONE, MD
Oregon Health \& Science University Portland, Oregon, USA

1 Doust J. Review: Vaginal signs and symptoms perform poorly in diagnosing vaginal candidiasis, bacterial vaginosis, and vaginal trichomoniasis. Evidence-Based Medicine 2004;9:153.

2 Nardone DA, Roth KM, Mazur DJ, et al. Usefulness of physical examination in detecting the presence or absence of anemia. Arch Intern Med 1990;150:201-4.

3 Landers DV, Wiesenfeld HC, Heine RP, et al. Predictive value of the clinical diagnosis of lower genital tract infection in women. Am J Obstet Gynecol 2004;190:1004-10.
We felt that lack of itching was reasonably good in eliminating candidiasis. Thus our formulation was that while symptoms were generally not good in distinguishing between the 3 causes, lack of itching made Candida less likely and lack of odour made BV less likely.

MATT ANDERSON, MD, MSC Albert Einstein College of Medicine Bronx, New York, USA

absence of symptoms and signs is even less helpful in ruling out disease, with only the absence of odour or yellow discharge ruling out BV."

1 Doust J. Review: Vaginal signs and symptoms perform poorly in diagnosing vaginal candidiasis, bacterial vaginosis, and vaginal trichomoniasis. Evidence-Based Medicine 2004;9:153. 